\title{
Heat Stability of Resistance to Southern Root-knot Nematode in Bell Pepper Genotypes Homozygous and Heterozygous for the $N$ Gene
}

\author{
Judy A. Thies ${ }^{1}$ and Richard L. Fery ${ }^{2}$ \\ U.S. Vegetable Laboratory, U.S. Department of Agriculture, Agricultural Research Service, 2875 \\ Savannah Highway, Charleston, SC 29414-5334
}

AdDitional INDEX wORDs. Capsicum annuum var. annuum, Meloidogyne incognita race 3, nematode resistance

\begin{abstract}
Expression of the $N$ gene, which confers resistance to southern root-knot nematode (Meloidogyne incognita Kofoid and White) in bell pepper [(Capsicum annuum L. var. annuum (Grossum Group)], is modified at high temperatures $\left(28{ }^{\circ} \mathrm{C}\right.$ and $\left.32{ }^{\circ} \mathrm{C}\right)$, but its expression in the heterozygous condition $(\mathrm{Nn})$ has not been documented at moderate or high temperatures. Responses of the near-isogenic bell pepper cultivars, Charleston Belle and Keystone Resistant Giant (differing at the $N$ locus), and the $F_{1}$ and reciprocal $F_{1}$ crosses between these cultivars to $M$. incognita race 3 were determined at 24,28 , and $32{ }^{\circ} \mathrm{C}$ in growth chamber experiments. 'Keystone Resistant Giant' (nn) was susceptible at 24,28 , and $32{ }^{\circ} \mathrm{C}$. 'Charleston Belle' $(N N)$ exhibited high resistance at $24{ }^{\circ} \mathrm{C}$ and resistance was partially lost at 28 and $32{ }^{\circ} \mathrm{C}$. However, at $32{ }^{\circ} \mathrm{C}$ root gall and egg mass severity indices for 'Charleston Belle' were still in the resistant range, and the number of $M$. incognita eggs per gram fresh root and reproductive index were $97 \%$ and $90 \%$ less, respectively, than for 'Keystone Resistant Giant'. Responses of the $F_{1}$ and $F_{1}$ reciprocal hybrid populations to $M$. incognita were similar to the response of the resistant parent at all temperatures. Root fresh weights and top dry weights indicated that both hybrid populations tolerated $M$. incognita infections at least as well as 'Charleston Belle'. These findings indicate that i) only one of the parental inbred lines needs to be converted to the $N N$ genotype to produce $F_{1}$ hybrid cultivars with fully functional $N$-type resistance to $M$. incognita; and ii) cytoplasmic factors are not involved in expression of $N$-type resistance and the resistant parental inbred can used to equal advantage as either the paternal or the maternal parent.
\end{abstract}

Resistance to the southern root-knot nematode (Meloidogyne incognita) in bell pepper (Capsicum annuum), is controlled by a single dominant gene designated $N$ by Hare (1957). Hare (1966) developed and released 'Mississippi Nemaheart', a pimiento pepper with resistance to $M$. incognita conditioned by the $N$ gene. Fery et al. (1998) transferred the $N$ gene from 'Mississippi Nemaheart' into 'Keystone Resistant Giant' and 'Yolo Wonder $\mathrm{B}$ ' bell pepper using recurrent backcrossing procedures. The products of this breeding effort were two $M$. incognita-resistant open-pollinated bell pepper cultivars, Charleston Belle and Carolina Wonder, that are homozygous resistant for the $N$ gene (Fery et al., 1998). Thies and Fery (1998) demonstrated that resistance of 'Charleston Belle' and 'Carolina Wonder' was compromised at 28 and $32{ }^{\circ} \mathrm{C}$. However, at $32{ }^{\circ} \mathrm{C}$, the root gall severity indices were still within the resistant range, and reproduction of $M$. incognita in both 'Charleston Belle' and 'Carolina Wonder' was only $20 \%$ of the reproduction in their respective susceptible recurrent parents, 'Keystone Resistant Giant' and 'Yolo Wonder B'. Thus, cultivars possessing resistance conferred by the $N$ gene should be of value to pepper producers in hot climates where $M$. incognita occurs.

None of the bell pepper cultivars currently available to United States growers have sufficient resistance to $M$. incognita (Fery et al., 1998). Root-knot nematode resistant cultivars such as 'Charleston Belle' or 'Carolina Wonder' that are homozygous $(N N)$ for the $N$ gene may be useful as parental lines for developing resistant

Received for publication 16 July 2001. Accepted for publication 17 Dec. 2001 The authors thank E.L. Corley, F.P. Maguire, S.W. Miller, S. Merrill, R. Reeves, and S. Ward for technical assistance in conducting the experiments. Mention of a trademark, proprietary product, or vendor does not constitute a guarantee or warranty of the product by the U.S. Dept. of Agriculture and does not imply its approval to the exclusion of other products or vendors that also may be suitable. ${ }^{1}$ Research plant pathologist.

${ }^{2}$ Research geneticist.
$\mathrm{F}_{1}$ hybrid $(\mathrm{Nn})$ bell pepper cultivars. The utility of $\mathrm{F}_{1}$ hybrids heterozygous for the $N$ gene will depend on expression of resistance to $M$. incognita, especially at high temperatures. However, expression of the $N$ gene in the heterozygous $(\mathrm{Nn})$ condition has not been evaluated at any temperature. Therefore, the objectives of this study were to 1) determine expression of resistance to $M$. incognita conferred by the homozygous $(N N)$ and heterozygous $\mathrm{N} \mathrm{Nn}$ ) conditions of the $\mathrm{N}$ locus at moderate and high temperatures, and 2) determine whether there are any cytoplasmic effects on expression of the $N$ gene in $\mathrm{F}_{1}$ bell pepper hybrids.

\section{Materials and Methods}

Nematode inoculum. A population of $M$. incognita race 3, obtained from S. A. Lewis, Clemson University, Clemson, S.C. was increased on 'Rutgers' tomato (Lycopersicon esculentum Mill.) and 'Kentucky Wonder 191' pole bean (Phaseolus vulgaris L.) in a greenhouse. Egg inocula for all experiments were extracted from infected tomato and pole bean roots using the $0.5 \% \mathrm{NaOCl}$ method (Hussey and Barker, 1973).

PePPER Genotypes. The bell pepper genotypes used in all experiments were 'Charleston Belle' $(\mathrm{CB}-N N)$, 'Keystone Resistant Giant' (KRG - nn), $\mathrm{F}_{1}(\mathrm{CB} \times \mathrm{KRG}-\mathrm{Nn})$, and $\mathrm{F}_{1}$ (KRG $\mathrm{xCB}-\mathrm{Nn}$ ). 'Charleston Belle' is an M. incognita-resistant, openpollinated bell pepper cultivar developed by scientists at the U.S. Vegetable Laboratory, U.S. Department of Agriculture, Agricultural Research Service (Fery et al., 1998). 'Charleston Belle' is homozygous for the $N$ gene that confers resistance to $M$. incognita, M. arenaria $(\mathrm{Neal})$ Chitwood races 1 and 2, and M.javanica (Treub) Chitwood (Fery et al., 1998; Thies and Fery, 2000a). 'Keystone Resistant Giant' $(n n)$ is the susceptible recurrent parental cultivar used in the backcross breeding procedure (six backcrosses) to develop 'Charleston Belle' (Fery et al., 1998). 'Charleston Belle' and 'Keystone Resistant Giant' were crossed 
to produce the $\mathrm{F}_{1}(\mathrm{CB} \times \mathrm{KRG}-\mathrm{Nn})$ and the reciprocal $\mathrm{F}_{1}(\mathrm{KRG}$ $\mathrm{x} \mathrm{CB}-\mathrm{Nn}$ ) populations.

GrowTH CHAMBER EXPERIMENTS. Reactions of 'Charleston Belle', 'Keystone Resistant Giant', $\mathrm{F}_{1}(\mathrm{CB} \times \mathrm{KRG}-\mathrm{Nn})$, and $\mathrm{F}_{1}$ $(\mathrm{KRG} \times \mathrm{CB}-\mathrm{Nn})$ to $M$. incognita race 3 were evaluated in separate experiments at each of three temperatures $(24,28$, and 32 $\left.{ }^{\circ} \mathrm{C}\right)$. The experimental design for each temperature experiment was a randomized complete block with 18 replications. Each replicate consisted of one plot (a pot containing a single plant) of each pepper genotype. Each temperature experiment was conducted three times, for a total of nine experiments.

Seeds of each pepper genotype were germinated in the greenhouse using plastic growing trays containing 50 individual 0.2L cells filled with Metro-Mix 360 (The Scotts Company, Marysville, Ohio). Three weeks later, seedlings were transplanted into individual 1-L pots filled with a pasteurized medium of 2 sandy loam soil : 1 washed river sand (by volume). About $5,000 \mathrm{M}$. incognita eggs in $5 \mathrm{~mL}$ distilled water were pipetted around the base of each plant at transplanting. Eighteen pots of each pepper genotype were placed in a growth chamber programmed to maintain the appropriate temperature (confirmed by measurement of soil and air temperatures). A 16-h photoperiod of $258 \mu \mathrm{mol} \cdot \mathrm{m}^{-2} \cdot \mathrm{s}^{-1}$ (photosynthetic active radiation, 400 to 760 $\mathrm{nm}$ ) was provided daily by fluorescent and incandescent lamps. Plants were fertilized 3 and 6 weeks after transplanting with $\mathrm{N}$ at $83 \mathrm{mg} \cdot \mathrm{L}^{-1}$ (2\% nitrate, $18 \%$ ammoniacal) from a $20 \mathrm{~N}-20 \mathrm{P}-16 \mathrm{~K}$ water soluble fertilizer (Peter's Fertilizer, United Industries Corporation, St. Louis, Mo.). Nine weeks after inoculation, the tops were severed at the crowns (stem-root junctions) of the plants, dried at $40{ }^{\circ} \mathrm{C}$ in a forced air oven for $\approx 2 \mathrm{~d}$, and top dry weights (DWs) recorded. The root system of each plant was removed from the soil, washed, and rated for severity of root galling and egg mass production using a scale of 1 to 5 where: 1 $=0 \%$ to $3 \%$ of the root system galled or covered with egg masses, $2=4 \%$ to $25 \%, 3=26 \%$ to $50 \%, 4=51 \%$ to $80 \%$, and $5=81 \%$ to $100 \%$ of the root system galled or covered with egg masses (Thies et al., 1998). Root gall and egg mass indices $\geq 3$ were considered susceptible reactions. Then, the entire root system was weighed and fresh weights (FWs) were recorded. The fibrous roots were clipped from the tap root, cut into 1 to $2-\mathrm{cm}$ pieces, and eggs were extracted from the entire sample of fibrous roots using 1.0\% $\mathrm{NaOCl}$ (Hussey and Barker, 1973). Eggs were counted using a stereomicroscope. Nematode reproduction was assessed by calculating a nematode reproduction factor (R) where $R=P_{f} / P_{i}$, with $P_{i}=$ the initial inoculum level and $P_{f}=$ the final egg recovery (Sasser et al., 1984).

Data ANALYSIS. Nematode egg and reproductive indices data were $\log _{10}(x+1)$ transformed to normalize the data before analysis (Noe, 1985). Data were analyzed using the general linear models (GLM) procedure of SAS for Windows (Statistical Analysis System, Version 6.12, SAS Inst. Inc., Cary, N.C.) and means were separated using Duncan's multiple range test at $P<0.05$.

Table 1. Gall indices, egg mass indices, numbers of Meloidogyne incognita eggs per gram fresh root, reproductive indices (RIs) of $M$. incognita, root fresh weights (FWs), and top dry weights (DWs) of Charleston Belle (CB), Keystone Resistant Giant (KRG), $\mathrm{F}_{1}(\mathrm{CB} \times \mathrm{KRG})$, and $\mathrm{F}_{1}(\mathrm{KRG}$ x CB) bell pepper inoculated with $M$. incognita race 3 and grown at $24{ }^{\circ} \mathrm{C} .{ }^{\mathrm{z}}$

\begin{tabular}{|c|c|c|c|c|c|c|}
\hline Genotype & $\begin{array}{c}\text { Gall } \\
\text { index }^{y}\end{array}$ & $\begin{array}{l}\text { Egg mass } \\
\text { index }^{y}\end{array}$ & $\begin{array}{l}\text { No. eggs/g } \\
\text { fresh root }\end{array}$ & $\mathrm{RI}^{\mathrm{x}}$ & $\begin{array}{l}\text { Root FW } \\
(\mathrm{g})\end{array}$ & $\begin{array}{c}\text { Top DW } \\
(\mathrm{g})\end{array}$ \\
\hline \multicolumn{7}{|l|}{ Test $1^{\mathrm{w}}$} \\
\hline Charleston Belle ${ }^{t}$ & $1.0 \mathrm{a}^{\mathrm{v}}$ & $1.0 \mathrm{a}$ & $114 \mathrm{a}^{\mathrm{u}}$ & $0.22 \mathrm{a}^{\mathrm{u}}$ & $7.49 \mathrm{ab}$ & $0.60 \mathrm{ab}$ \\
\hline Keystone Resistant Giant ${ }^{\mathrm{s}}$ & $3.1 \mathrm{~b}$ & $2.9 \mathrm{~b}$ & $14,155 \mathrm{~b}$ & $26.19 \mathrm{~b}$ & $6.69 \mathrm{a}$ & $0.42 \mathrm{a}$ \\
\hline $\mathrm{F}_{1}(\mathrm{CB} \times \mathrm{KRG})$ & $1.0 \mathrm{a}$ & $1.0 \mathrm{a}$ & $106 \mathrm{a}$ & $0.24 \mathrm{a}$ & $8.22 \mathrm{~b}$ & $0.63 \mathrm{c}$ \\
\hline $\mathrm{F}_{1}(\mathrm{KRG} \times \mathrm{CB})$ & $1.0 \mathrm{a}$ & $1.0 \mathrm{a}$ & $151 \mathrm{a}$ & $0.29 \mathrm{a}$ & $6.85 \mathrm{a}$ & $0.49 \mathrm{ab}$ \\
\hline \multicolumn{7}{|l|}{ Test $2^{\mathrm{w}}$} \\
\hline Charleston Belle & $1.0 \mathrm{a}$ & $1.0 \mathrm{a}$ & $15 \mathrm{a}$ & $0.01 \mathrm{a}$ & $4.69 \mathrm{a}$ & $0.49 \mathrm{a}$ \\
\hline Keystone Resistant Giant & $2.9 \mathrm{~b}$ & $2.6 \mathrm{~b}$ & $7,963 \mathrm{~b}$ & $11.31 \mathrm{~b}$ & $5.39 \mathrm{a}$ & $0.57 \mathrm{a}$ \\
\hline $\mathrm{F}_{1}(\mathrm{CB} \times \mathrm{KRG})$ & $1.0 \mathrm{a}$ & $1.0 \mathrm{a}$ & $26 \mathrm{a}$ & $0.03 \mathrm{a}$ & $4.89 \mathrm{a}$ & $0.53 \mathrm{a}$ \\
\hline $\mathrm{F}_{1}(\mathrm{KRG} \times \mathrm{CB})$ & $1.0 \mathrm{a}$ & $1.0 \mathrm{a}$ & $14 \mathrm{a}$ & $0.02 \mathrm{a}$ & $5.41 \mathrm{a}$ & $0.56 \mathrm{a}$ \\
\hline \multicolumn{7}{|l|}{ Test $3^{\mathrm{w}}$} \\
\hline Charleston Belle & $1.0 \mathrm{a}$ & $1.0 \mathrm{a}$ & $130 \mathrm{a}$ & $0.07 \mathrm{a}$ & $2.25 \mathrm{a}$ & $0.19 \mathrm{a}$ \\
\hline Keystone Resistant Giant & $4.0 \mathrm{~b}$ & $3.7 \mathrm{~b}$ & $13,228 \mathrm{~b}$ & $3.48 \mathrm{~b}$ & $1.30 \mathrm{~b}$ & $0.10 \mathrm{~b}$ \\
\hline $\mathrm{F}_{1}(\mathrm{CB} \times \mathrm{KRG})$ & $1.0 \mathrm{a}$ & $1.0 \mathrm{a}$ & $130 \mathrm{a}$ & $0.08 \mathrm{a}$ & $2.71 \mathrm{a}$ & $0.21 \mathrm{a}$ \\
\hline $\mathrm{F}_{1}(\mathrm{KRG} \times \mathrm{CB})$ & $1.0 \mathrm{a}$ & $1.0 \mathrm{a}$ & $117 \mathrm{a}$ & $0.06 \mathrm{a}$ & $2.30 \mathrm{a}$ & $0.18 \mathrm{a}$ \\
\hline \multicolumn{7}{|l|}{ Combined analysis of all tests } \\
\hline Charleston Belle & $1.0 \mathrm{a}$ & $1.0 \mathrm{a}$ & $61 \mathrm{a}$ & $0.06 \mathrm{a}$ & $4.81 \mathrm{a}$ & $0.42 \mathrm{a}$ \\
\hline Keystone Resistant Giant & $3.3 \mathrm{~b}$ & $3.1 \mathrm{~b}$ & $11,424 \mathrm{~b}$ & $10.10 \mathrm{~b}$ & $4.46 \mathrm{~b}$ & $0.36 \mathrm{~b}$ \\
\hline $\mathrm{F}_{1}(\mathrm{CB} \times \mathrm{KRG})$ & $1.0 \mathrm{a}$ & $1.0 \mathrm{a}$ & $71 \mathrm{a}$ & $0.08 \mathrm{a}$ & $5.27 \mathrm{a}$ & $0.45 \mathrm{a}$ \\
\hline $\mathrm{F}_{1}(\mathrm{KRG} \times \mathrm{CB})$ & $1.0 \mathrm{a}$ & $1.0 \mathrm{a}$ & $57 \mathrm{a}$ & $0.06 \mathrm{a}$ & $4.65 \mathrm{~b}$ & $0.40 \mathrm{ab}$ \\
\hline
\end{tabular}

${ }_{\overline{\mathrm{z}} \text { Each plant was inoculated with } \approx 5000 \text { eggs at transplanting. Results of combined analyses of the three tests indicated a significant pepper genotype }}$ $\times$ test interaction for all variables. Consequently, results are presented for both single test and combined analyses to aid interpretation.

${ }^{y}$ Scale of 1 to 5 , where $1=0 \%$ to $3 \%$ root system galled or covered with egg masses, $2=4 \%$ to $25 \%, 3=26 \%$ to $50 \%, 4=51 \%$ to $80 \%$, and $5=$ $81 \%$ to $100 \%$ root system galled or covered with egg masses.

${ }^{\times} \mathrm{RI}=$ final egg recovery/initial inoculum level of M. incognita, 9 weeks after inoculation.

${ }^{\mathrm{w}}$ Data are means of 18 single plant replications.

vMean separation within a column and test by Duncan's multiple range test, $P<0.05$.

"Data were $\log _{10}(\mathrm{x}+1)$ transformed before analysis. Back transformed data are shown.

tHomozygous dominant $(N N)$ for resistance to $M$. incognita.

${ }^{\text {s}}$ Susceptible to $M$. incognita. 


\section{Results}

The near-isogenic lines 'Charleston Belle' and 'Keystone Resistant Giant' reacted as expected. 'Charleston Belle' exhibited high resistance at $24^{\circ} \mathrm{C}$ (Table 1) and resistance [as measured by gall (GI) and egg mass (EMI) indices, numbers of eggs per gram fresh root, and reproductive index (RI)] was partially lost at 28 and $32{ }^{\circ} \mathrm{C}$ (Tables 2 and 3). 'Keystone Resistant Giant' was susceptible at all three temperatures, but exhibited highly susceptible reactions at 28 and $32{ }^{\circ} \mathrm{C}$. Generally, the reactions of the $\mathrm{F}_{1}(\mathrm{CB} \times \mathrm{KRG})$ and $\mathrm{F}_{1}(\mathrm{KRG} \times \mathrm{CB})$ hybrid populations were similar to the reaction of the root-knot resistant parent, 'Charleston Belle'. Results of combined analyses of the three tests within each temperature study $\left(24,28\right.$, and $\left.32^{\circ} \mathrm{C}\right)$ indicated a significant pepper genotype $\times$ test interaction for all variables. Results are presented in the tables for both single test and combined analyses to aid interpretation, but the discussion of results below is limited to the combined analyses.

Test at $24^{\circ} \mathrm{C}$. 'Charleston Belle' exhibited high resistance at $24^{\circ} \mathrm{C}$ (Table 1). For example, results of the combined analysis of all tests indicated there was no visible galling and very minimal egg mass production on roots of 'Charleston Belle', and the numbers of $M$. incognita eggs per gram fresh root and reproductive index for 'Charleston Belle' were both $99 \%$ lower $(P<0.05)$ than for 'Keystone Resistant Giant' (Table 1). Both of the hybrids, $\mathrm{F}_{1}(\mathrm{CB} \times \mathrm{KRG})$ and $\mathrm{F}_{1}(\mathrm{KRG} \times \mathrm{CB})$, exhibited high resistance to $M$. incognita similar to that exhibited by 'Charleston Belle'. For instance, root galling and egg mass production were undetectable for both $\mathrm{F}_{1}$ hybrids [(GI $=1.0$ and $\mathrm{EMI}=1.0$ for $F_{1}(C B \times K R G) ; G I=1.0$ and $E M I=1.0$ for $\left.F_{1}(K R G \times C B)\right]$. Numbers of eggs per gram fresh root for both $F_{1}$ hybrids were $<100$ and the reproductive indices were $<0.1$, indicating that both $F_{1}$ hybrids were highly resistant. Root FWs of 'Charleston Belle' and $\mathrm{F}_{1}(\mathrm{CB} \times \mathrm{KRG})$ were $8 \%$ and $18 \%$ heavier $(P<0.05)$, respectively, than root FW of 'Keystone Resistant Giant'. Likewise, top DWs of 'Charleston Belle' and $\mathrm{F}_{1}(\mathrm{CB} \times \mathrm{KRG})$ were $17 \%$ and $25 \%$ heavier $(P<0.05)$, respectively, than the top DW of 'Keystone Resistant Giant'. At $24^{\circ} \mathrm{C}$, resistance of the $\mathrm{F}_{1}(\mathrm{CB}$ $\mathrm{x} K R G)$ and $F_{1}(\mathrm{KRG} \times \mathrm{CB})$ appeared to be similar to that expressed by the homozygous resistant parent 'Charleston Belle'.

Test at $28{ }^{\circ} \mathbf{C}$. 'Charleston Belle' exhibited moderate resistance at $28^{\circ} \mathrm{C}$, i.e., the root gall index and egg mass index means from the combined analysis were low $(\mathrm{GI}=1.6 ; \mathrm{EMI}=1.6)$ and the numbers of $M$. incognita eggs per gram fresh root and reproductive index were $95 \%$ and $93 \%$ less $(P<0.05)$, respectively, than for 'Keystone Resistant Giant' (Table 2). 'Keystone Resistant Giant' was highly susceptible (GI and EMI $=4.1 ; \mathrm{RI}=$ 34.7). The $F_{1}(C B \times K R G)$ and $F_{1}(K R G \times C B)$ hybrids exhibited moderate resistance to $M$. incognita and their reactions were similar to those of 'Charleston Belle'. Root FW for 'Charleston Belle', $\mathrm{F}_{1}(\mathrm{CB} \times \mathrm{KRG})$, and $\mathrm{F}_{1}(\mathrm{KRG} \times \mathrm{CB})$ (average of the three genotypes) was $33 \%$ heavier $(P<0.05)$ than that for 'Keystone

Table 2. Gall indices, egg mass indices, numbers of Meloidogyne incognita eggs per gram fresh root, reproductive indices (RIs) of $M$. incognita, root fresh weights (FWs), and top dry weights (DWs) of Charleston Belle (CB), Keystone Resistant Giant $(\mathrm{KRG}), \mathrm{F}_{1}(\mathrm{CB} \times \mathrm{KRG})$, and $\mathrm{F}_{1}(\mathrm{KRG}$ x CB) bell pepper inoculated with $M$. incognita race 3 and grown at $28^{\circ} \mathrm{C}{ }^{\mathrm{z}}$

\begin{tabular}{|c|c|c|c|c|c|c|}
\hline Genotype & $\begin{array}{c}\text { Gall } \\
\text { index }{ }^{y}\end{array}$ & $\begin{array}{c}\text { Egg mass } \\
\text { index }^{y}\end{array}$ & $\begin{array}{l}\text { No. eggs/g } \\
\text { fresh root }\end{array}$ & $\mathrm{RI}^{\mathrm{x}}$ & $\begin{array}{l}\text { Root FW } \\
(\mathrm{g})\end{array}$ & $\begin{array}{c}\text { Top DW } \\
\text { (g) }\end{array}$ \\
\hline \multicolumn{7}{|l|}{ Test $1^{\mathrm{w}}$} \\
\hline Charleston Belle ${ }^{t}$ & $2.2 \mathrm{~b}^{\mathrm{v}}$ & $2.2 \mathrm{~b}$ & $10,247 b^{u}$ & $13.20 \mathrm{~b}^{\mathrm{u}}$ & $4.55 \mathrm{~b}$ & $0.42 \mathrm{~b}$ \\
\hline Keystone Resistant Giant ${ }^{\mathrm{s}}$ & $3.8 \mathrm{c}$ & $4.0 \mathrm{c}$ & $47,566 \mathrm{c}$ & $39.61 \mathrm{~b}$ & $3.06 \mathrm{a}$ & $0.32 \mathrm{a}$ \\
\hline $\mathrm{F}_{1}(\mathrm{CB} \times \mathrm{KRG})$ & $1.8 \mathrm{ab}$ & $1.8 \mathrm{ab}$ & $9,759 \mathrm{~b}$ & $15.15 \mathrm{~b}$ & $5.34 \mathrm{c}$ & $0.48 \mathrm{~b}$ \\
\hline $\mathrm{F}_{1}(\mathrm{KRG} \times \mathrm{CB})$ & $1.6 \mathrm{a}$ & $1.6 \mathrm{a}$ & $2,086 \mathrm{a}$ & $2.93 \mathrm{a}$ & $4.97 \mathrm{bc}$ & $0.48 \mathrm{~b}$ \\
\hline \multicolumn{7}{|l|}{ Test $2^{\mathrm{w}}$} \\
\hline Charleston Belle & $1.2 \mathrm{a}$ & $1.2 \mathrm{a}$ & $312 \mathrm{a}$ & $0.40 \mathrm{a}$ & $4.63 \mathrm{a}$ & $0.43 \mathrm{a}$ \\
\hline Keystone Resistant Giant & $3.8 \mathrm{~b}$ & $3.7 \mathrm{~b}$ & $41,058 \mathrm{~b}$ & $46.14 \mathrm{~b}$ & $4.54 \mathrm{a}$ & $0.47 \mathrm{a}$ \\
\hline $\mathrm{F}_{1}(\mathrm{CB} \times \mathrm{KRG})$ & $1.4 \mathrm{a}$ & $1.3 \mathrm{a}$ & $725 \mathrm{a}$ & $1.01 \mathrm{a}$ & $5.02 \mathrm{a}$ & $0.51 \mathrm{a}$ \\
\hline $\mathrm{F}_{1}(\mathrm{KRG} \times \mathrm{CB})$ & $1.1 \mathrm{a}$ & $1.5 \mathrm{a}$ & $832 \mathrm{a}$ & $1.16 \mathrm{a}$ & $5.65 \mathrm{~b}$ & $0.51 \mathrm{a}$ \\
\hline \multicolumn{7}{|l|}{ Test $3^{\mathrm{w}}$} \\
\hline Charleston Belle & $1.5 \mathrm{a}$ & $1.5 \mathrm{a}$ & $2,425 \mathrm{a}$ & $2.10 \mathrm{a}$ & $3.47 \mathrm{~b}$ & $0.29 \mathrm{~b}$ \\
\hline Keystone Resistant Giant & $4.8 \mathrm{~b}$ & $4.7 \mathrm{~b}$ & $41,371 \mathrm{~b}$ & $22.81 \mathrm{~b}$ & $2.51 \mathrm{a}$ & $0.21 \mathrm{a}$ \\
\hline $\mathrm{F}_{1}(\mathrm{CB} \times \mathrm{KRG})$ & $1.6 \mathrm{a}$ & $1.6 \mathrm{a}$ & $2,399 \mathrm{a}$ & $2.18 \mathrm{a}$ & $3.59 \mathrm{~b}$ & $0.31 \mathrm{~b}$ \\
\hline $\mathrm{F}_{1}(\mathrm{KRG} \times \mathrm{CB})$ & $1.5 \mathrm{a}$ & $1.5 \mathrm{a}$ & $2,184 \mathrm{a}$ & $1.85 \mathrm{a}$ & $3.39 \mathrm{~b}$ & $0.31 \mathrm{~b}$ \\
\hline \multicolumn{7}{|l|}{ Combined analysis of all tests } \\
\hline Charleston Belle & $1.6 \mathrm{a}$ & $1.6 \mathrm{a}$ & $2,050 \mathrm{a}$ & $2.30 \mathrm{a}$ & $4.22 \mathrm{~b}$ & $0.38 \mathrm{ab}$ \\
\hline Keystone Resistant Giant & $4.1 \mathrm{~b}$ & $4.1 \mathrm{~b}$ & $43,271 \mathrm{~b}$ & $34.67 \mathrm{~b}$ & $3.37 \mathrm{a}$ & $0.34 \mathrm{a}$ \\
\hline $\mathrm{F}_{1}(\mathrm{CB} \times \mathrm{KRG})$ & $1.6 \mathrm{a}$ & $1.6 \mathrm{a}$ & $2,508 \mathrm{a}$ & $3.15 \mathrm{a}$ & $4.65 \mathrm{~b}$ & $0.43 \mathrm{c}$ \\
\hline $\mathrm{F}_{1}(\mathrm{KRG} \times \mathrm{CB})$ & $1.6 \mathrm{a}$ & $1.5 \mathrm{a}$ & $1,513 \mathrm{a}$ & $1.76 \mathrm{a}$ & $4.60 \mathrm{~b}$ & $0.42 \mathrm{bc}$ \\
\hline
\end{tabular}

${ }^{\mathrm{z}}$ Each plant was inoculated with $\approx 5000$ eggs at transplanting. Results of combined analyses of the three tests indicated a significant pepper genotype $\times$ test interaction for all variables. Consequently, results are presented for both single test and combined analyses to aid interpretation.

${ }^{y}$ Scale of 1 to 5 , where $1=0 \%$ to $3 \%$ root system galled or covered with egg masses, $2=4 \%$ to $25 \%, 3=26 \%$ to $50 \%, 4=51 \%$ to $80 \%$, and $5=$ $81 \%$ to $100 \%$ root system galled or covered with egg masses.

${ }^{\mathrm{x}} \mathrm{RI}=$ final egg recovery/initial inoculum level of $M$. incognita, 9 weeks after inoculation.

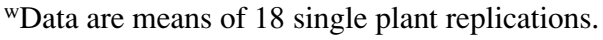

'Mean separation within a column and test by Duncan's multiple range test, $P<0.05$.

uData were $\log _{10}(\mathrm{x}+1)$ transformed before analysis. Back transformed data are shown.

tHomozygous dominant $(N N)$ for resistance to $M$. incognita.

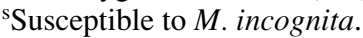


Table 3. Gall indices, egg mass indices, numbers of Meloidogyne incognita eggs per gram fresh root, reproductive indices (RIs) of M. incognita, root fresh weights (FWs), and top dry weights (DWs) of Charleston Belle (CB), Keystone Resistant Giant $(\mathrm{KRG}), \mathrm{F}_{1}(\mathrm{CB} \times \mathrm{KRG})$, and $\mathrm{F}_{1}(\mathrm{KRG}$ x CB) bell pepper inoculated with $M$. incognita race 3 and grown at $32{ }^{\circ} \mathrm{C} .{ }^{\mathrm{z}}$

\begin{tabular}{|c|c|c|c|c|c|c|}
\hline Genotype & $\begin{array}{c}\text { Gall } \\
\text { index }\end{array}$ & $\begin{array}{l}\text { Egg mass } \\
\text { index }^{y}\end{array}$ & $\begin{array}{l}\text { No. eggs/g } \\
\text { fresh root }\end{array}$ & $\mathrm{RI}^{\mathrm{x}}$ & $\begin{array}{l}\text { Root FW } \\
\text { (g) }\end{array}$ & $\begin{array}{c}\text { Top DW } \\
\text { (g) }\end{array}$ \\
\hline \multicolumn{7}{|l|}{ Test $1^{\mathrm{w}}$} \\
\hline Charleston Belle $^{\mathrm{t}}$ & $3.2 \mathrm{a}^{\mathrm{v}}$ & $3.3 \mathrm{a}$ & $26,248 \mathrm{a}^{\mathrm{u}}$ & $38.05 \mathrm{a}^{\mathrm{u}}$ & $5.07 \mathrm{~b}$ & $0.62 \mathrm{ab}$ \\
\hline Keystone Resistant Giant ${ }^{\mathrm{s}}$ & $4.5 \mathrm{~b}$ & $4.7 \mathrm{~b}$ & $172,227 \mathrm{c}$ & $126.33 \mathrm{~b}$ & $3.09 \mathrm{a}$ & $0.52 \mathrm{a}$ \\
\hline $\mathrm{F}_{1}(\mathrm{CB} \times \mathrm{KRG})$ & $3.6 \mathrm{a}$ & $3.7 \mathrm{a}$ & $78,868 \mathrm{bc}$ & $139.89 \mathrm{~b}$ & $6.22 \mathrm{c}$ & $0.72 \mathrm{~b}$ \\
\hline $\mathrm{F}_{1}(\mathrm{KRG} \times \mathrm{CB})$ & $3.3 \mathrm{a}$ & $3.2 \mathrm{a}$ & $37,653 \mathrm{ab}$ & $58.90 \mathrm{ab}$ & $5.60 \mathrm{bc}$ & $0.72 \mathrm{~b}$ \\
\hline \multicolumn{7}{|l|}{ Test $2^{\mathrm{w}}$} \\
\hline Charleston Belle & $1.4 \mathrm{a}$ & $1.4 \mathrm{a}$ & $517 \mathrm{a}$ & $0.47 \mathrm{a}$ & $3.39 \mathrm{c}$ & $0.65 \mathrm{~b}$ \\
\hline Keystone Resistant Giant & $4.8 \mathrm{~b}$ & $4.8 \mathrm{~b}$ & $219,483 \mathrm{c}$ & $33.22 \mathrm{~b}$ & $0.68 \mathrm{a}$ & $0.11 \mathrm{a}$ \\
\hline $\mathrm{F}_{1}(\mathrm{CB} \times \mathrm{KRG})$ & $1.6 \mathrm{a}$ & $1.6 \mathrm{a}$ & $1,680 \mathrm{~b}$ & $1.11 \mathrm{a}$ & $2.64 \mathrm{~b}$ & $0.62 \mathrm{a}$ \\
\hline $\mathrm{F}_{1}(\mathrm{KRG} \times \mathrm{CB})$ & $1.7 \mathrm{a}$ & $1.5 \mathrm{a}$ & $758 \mathrm{ab}$ & $0.65 \mathrm{a}$ & $3.09 \mathrm{bc}$ & $0.69 \mathrm{~b}$ \\
\hline \multicolumn{7}{|l|}{ Test $3^{\mathrm{w}}$} \\
\hline Charleston Belle & $2.7 \mathrm{a}$ & $2.7 \mathrm{a}$ & $14,866 \mathrm{a}$ & $12.07 \mathrm{a}$ & $4.17 \mathrm{~b}$ & $0.42 \mathrm{c}$ \\
\hline Keystone Resistant Giant & $5.0 \mathrm{~b}$ & $5.0 \mathrm{~b}$ & $148,970 \mathrm{~b}$ & $52.88 \mathrm{~b}$ & $1.81 \mathrm{a}$ & $0.11 \mathrm{a}$ \\
\hline $\mathrm{F}_{1}(\mathrm{CB} \times \mathrm{KRG})$ & $2.6 \mathrm{a}$ & $2.7 \mathrm{a}$ & $9,460 \mathrm{a}$ & $9.24 \mathrm{a}$ & $3.78 \mathrm{a}$ & $0.33 \mathrm{~b}$ \\
\hline $\mathrm{F}_{1}(\mathrm{KRG} \times \mathrm{CB})$ & $2.9 \mathrm{a}$ & $3.1 \mathrm{a}$ & $17,567 \mathrm{a}$ & $16.78 \mathrm{ab}$ & $3.85 \mathrm{a}$ & $0.37 \mathrm{bc}$ \\
\hline \multicolumn{7}{|l|}{ Combined analysis of all tests } \\
\hline Charleston Belle & $2.4 \mathrm{a}$ & $2.5 \mathrm{a}$ & $5,657 \mathrm{a}$ & $5.94 \mathrm{a}$ & $4.21 \mathrm{~b}$ & $0.56 \mathrm{~b}$ \\
\hline Keystone Resistant Giant & $4.8 \mathrm{~b}$ & $4.8 \mathrm{~b}$ & $178,525 \mathrm{~b}$ & $60.70 \mathrm{~b}$ & $1.86 \mathrm{a}$ & $0.19 \mathrm{a}$ \\
\hline $\mathrm{F}_{1}(\mathrm{CB} \times \mathrm{KRG})$ & $2.6 \mathrm{a}$ & $2.7 \mathrm{a}$ & $11,202 \mathrm{a}$ & $11.83 \mathrm{a}$ & $4.24 \mathrm{~b}$ & $0.56 \mathrm{~b}$ \\
\hline $\mathrm{F}_{1}(\mathrm{KRG} \times \mathrm{CB})$ & $2.6 \mathrm{a}$ & $2.6 \mathrm{a}$ & 7,945 a & $8.62 \mathrm{a}$ & $4.18 \mathrm{~b}$ & $0.58 \mathrm{~b}$ \\
\hline
\end{tabular}

${ }^{\mathrm{z}}$ Each plant was inoculated with $\approx 5000$ eggs at transplanting. Results of combined analyses of the three tests indicated a significant pepper genotype $\times$ test interaction for all variables. Consequently, results are presented for both single test and combined analyses to aid interpretation.

${ }^{y}$ Scale of 1 to 5 , where $1=0 \%$ to $3 \%$ root system galled or covered with egg masses, $2=4 \%$ to $25 \%, 3=26 \%$ to $50 \%, 4=51 \%$ to $80 \%$, and $5=$ $81 \%$ to $100 \%$ root system galled or covered with egg masses.

${ }^{\mathrm{x}} \mathrm{RI}=$ final egg recovery/initial inoculum level of $M$. incognita, 9 weeks after inoculation.

wData are means of 18 single plant replications.

vMean separation within a column and test by Duncan's multiple range test, $P<0.05$.

"Data were $\log _{10}(\mathrm{x}+1)$ transformed before analysis. Back transformed data are shown.

tHomozygous dominant $(N N)$ for resistance to $M$. incognita.

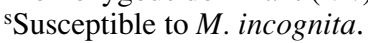

Resistant Giant'. Top DWs of both $\mathrm{F}_{1}$ hybrids were $26 \%$ heavier $(P<0.05)$ than that for 'Keystone Resistant Giant'. At $28{ }^{\circ} \mathrm{C}$, expression of resistance conferred by the $N$ gene appeared to be as effective in the heterozygous $F_{1}(\mathrm{CB} \times \mathrm{KRG})$ and $\mathrm{F}_{1}(\mathrm{KRG} \mathrm{x}$ $\mathrm{CB}$ ) hybrids as in the homozygous resistant parent 'Charleston Belle'.

Test at $32{ }^{\circ} \mathbf{C}$. 'Charleston Belle' exhibited low resistance at $32^{\circ} \mathrm{C}$; i. e., root gall index and egg mass index means from the combined analysis were intermediate $(\mathrm{GI}=2.4 ; \mathrm{EMI}=2.5)$ and the number of $M$. incognita eggs per gram fresh root and reproductive index were $97 \%$ and $90 \%$ less $(P<0.05)$, respectively, than for 'Keystone Resistant Giant' (Table 3). 'Keystone Resistant Giant' was extremely susceptible $(\mathrm{GI}=4.8 ; \mathrm{EMI}=4.8 ; \mathrm{RI}=$ 60.70). The $\mathrm{F}_{1}(\mathrm{CB} \times \mathrm{KRG})$ and $\mathrm{F}_{1}(\mathrm{KRG} \times \mathrm{CB})$ hybrids exhibited low resistance to $M$. incognita at $32{ }^{\circ} \mathrm{C}$; the severity of root galling, amount of egg mass production, numbers of eggs per gram fresh root, and reproduction of $M$. incognita were similar to those of 'Charleston Belle'. Root FW for 'Charleston Belle', $\mathrm{F}_{1}(\mathrm{CB} \times \mathrm{KRG})$, and $\mathrm{F}_{1}(\mathrm{KRG} \times \mathrm{CB})$ (average of the three genotypes) was $126 \%$ heavier $(P<0.05)$ than for 'Keystone Resistant Giant'; also, top DW of 'Charleston Belle' and both $\mathrm{F}_{1}$ hybrids (average of the three genotypes) was 198\% heavier $(P \leq 0.05)$ than for 'Keystone Resistant Giant'. Differences between the $F_{1}$ hybrids and 'Charleston Belle' were not detectable for root FWs or top DWs. At $32{ }^{\circ} \mathrm{C}$, expression of resistance in $\mathrm{F}_{1}(\mathrm{CB} \times \mathrm{KRG})$ and $\mathrm{F}_{1}(\mathrm{KRG} \times \mathrm{CB})$ appeared to equal that of 'Charleston Belle'.

\section{Discussion}

'Charleston Belle' exhibited high resistance at $24{ }^{\circ} \mathrm{C}$ and resistance was partially lost at 28 and $32{ }^{\circ} \mathrm{C}$, confirming results of previous studies (Thies and Fery, 1998; 2000b). 'Keystone Resistant Giant' was susceptible at all three temperatures, but was extremely susceptible at $28{ }^{\circ} \mathrm{C}$ and $32{ }^{\circ} \mathrm{C}$; these results were also similar to our previous studies (Thies and Fery, 1998; Thies and Fery, 2000b). Both the $\mathrm{F}_{1}(\mathrm{CB} \times \mathrm{KRG})$ and $\mathrm{F}_{1}(\mathrm{KRG} \times \mathrm{CB})$ exhibited high resistance to $M$. incognita at $24{ }^{\circ} \mathrm{C}$, moderate resistance at 28 ${ }^{\circ} \mathrm{C}$, and low resistance at $32{ }^{\circ} \mathrm{C}$, similar in reactions to their homozygous resistant parent 'Charleston Belle'. This is the first report of expression of resistance to $M$. incognita in $\mathrm{F}_{1}$ bell pepper hybrids heterozygous for the $N$ gene at moderate and high temperatures.

Root $\mathrm{FWs}$ of $\mathrm{F}_{1}(\mathrm{CB} \times \mathrm{KRG})$ and $\mathrm{F}_{1}(\mathrm{KRG} \times \mathrm{CB})$ (mean of both genotypes) were $37 \%$ and $126 \%$ heavier than root FWs of 'Keystone Resistant Giant' at $28^{\circ} \mathrm{C}$ and $32^{\circ} \mathrm{C}$, respectively. Top DWs of $\mathrm{F}_{1}(\mathrm{CB} \times \mathrm{KRG})$ and $\mathrm{F}_{1}$ (KRG x CB) (mean of both genotypes) were $26 \%$ and $200 \%$ heavier than for 'Keystone Resistant Giant'. Root FWs and top DWs of $F_{1}(C B \times K R G)$ and $F_{1}(K R G \times C B)$ were equal to or significantly greater than 'Charleston Belle' at both 28 and $32{ }^{\circ} \mathrm{C}$. These data indicate that the $\mathrm{F}_{1}$ hybrids were remarkably more tolerant to $M$. incognita infection at high temperatures than the susceptible 'Keystone Resistant Giant' and that these hybrids also tolerate such infection at least as well as 'Charleston Belle'. 
Our results are similar to those of Abdul-Baki and Haroon (1996) who reported that root explants of tomato genotypes homozygous and heterozygous for the $M i$ gene that controls resistance to $M$. incognita were equally resistant at 28 and $31^{\circ} \mathrm{C}$, but both the homozygous and heterozygous genotypes partially lost resistance at $34{ }^{\circ} \mathrm{C}$. Resistance of both the homozygous and heterozygous tomato genotypes broke down at $37{ }^{\circ} \mathrm{C}$ (AbdulBaki and Haroon, 1996). Cap et al. (1993) reported that heat stable resistance conferred by the completely dominant $M i-2$ gene in a wild species of tomato [L. peruvianum (L.) Mill.] was equally effective and stable in the resistant parental plants and the $\mathrm{F}_{1}$ heterozygous progeny at both $25^{\circ} \mathrm{C}$ and $30^{\circ} \mathrm{C}$.

Resistance to $M$. incognita (isolate origin: Calissane, France) controlled by the single dominant $M e 3$ gene was stable in both the parental pepper (C. annuum) line PM687 (U.S. Plant Introduction 322719 ) and the $F_{1}$ hybrid PM687 x 'Yolo Wonder' at 32 and 42 ${ }^{\circ} \mathrm{C}$ (Djian-Caporalino et al., 1999). However, plants were subjected to temperatures of $32{ }^{\circ} \mathrm{C}$ for 1 week before and 3 weeks following inoculation with $500 \mathrm{M}$. incognita second-stage juveniles. Then, the plants were maintained at $22{ }^{\circ} \mathrm{C}$ for an additional 4 -week period to allow completion of one nematode generation. In the present experiment, resistance conferred by the $N$ gene in both homozygous and heterozygous $C$. annuum genotypes was compromised when plants were grown at $32{ }^{\circ} \mathrm{C}$ for 9 weeks following inoculation with 5,000 eggs of $M$. incognita race 3 . Differences in results between the present study of heat stability of expression of the $N$ gene and that of the Me3 gene (DjianCaporalino et al., 1999) may be due to differences in $M$. incognita isolates, life stages and levels of $M$. incognita inoculum, $C$. annuum genetic systems, and/or heat treatment regimes.

Results of the present study indicate that open-pollinated bell pepper lines homozygous at the $N$ locus for the dominant $N$ allele are readily useful as parental lines to develop southern root-knot nematode-resistant $F_{1}$ hybrids. Near-isogenic lines exhibited similar levels of resistance at 24,28 , or $32{ }^{\circ} \mathrm{C}$ regardless of whether the $N$ gene was in the homozygous $(N N)$ or heterozygous $(\mathrm{Nn})$ condition. 'Charleston Belle' and the heterozygous $\mathrm{F}_{1}$ hybrids exhibited high resistance at $24{ }^{\circ} \mathrm{C}$ and the resistance was partially lost at 28 and $32{ }^{\circ} \mathrm{C}$. However, at $32^{\circ} \mathrm{C}$, the root gall and egg mass severity indices for the three resistant near-isogenic lines were still in the low resistance range, and the number of $M$. incognita eggs per gram fresh root and reproductive index were 95\% and $85 \%$ less, respectively, than for 'Keystone Resistant Giant'. Additionally, the level of resistance exhibited at any given temperature by a heterozygous resistant $\mathrm{F}_{1}$ hybrid was independent of whether the resistant parental line was used as the paternal or maternal parent, and this indicates that cytoplasmic factors are not involved in conditioning $N$-type resistance. These findings provide plant breeders with two types of information needed to devise efficient and effective procedures for developing southern root-knot nematode resistant $\mathrm{F}_{1}$ hybrid bell pepper cultivars. First, they will need to convert only one of the parental lines to the $N N$ genotype to produce an $\mathrm{F}_{1}$ hybrid with fully functional $N$-type resistance. Second, the homozygous resistant parental line $(N N)$ can be used to equal advantage as either a paternal parent or a maternal parent.

\section{Literature Cited}

Abdul-Baki, A.A. and S.A. Haroon. 1996. Temperature effects on resistance to Meloidogyne sp. in excised tomato roots. HortScience 31:147-149.

Cap, G.B., P.A. Roberts, and I.J. Thomason. 1993. Inheritance of heatstable resistance to Meloidogyne incognita in Lycopersicon peruvianum and its relationship to the $M i$ gene. Theor. Appl. Genet. 85:777-783.

Djian-Caporalino, C., L. Pijarowski, A. Januel, V.Lefebvre, A. Daubèze, A. Palloix, A. Dalmasso, and P. Abad. 1999. Spectrum of resistance to root-knot nematodes and inheritance of heat-stable resistance in pepper (Capsicum annuum L.). Theor. Appl. Genet. 99:496-502.

Fery, R.L., P.D. Dukes, Sr., and J.A. Thies. 1998. 'Carolina Wonder' and 'Charleston Belle': Southern root-knot nematode resistant bell peppers. HortScience 33:900-902.

Hare, W.W. 1957. Inheritance of resistance to root-knot nematodes in pepper. Phytopathology 47:455-459.

Hare, W.W. 1966. New pimiento is resistant to nematodes. Miss. Farm Res. 29(2):1, 8.

Hussey, R.S. and K.R. Barker. 1973. A comparison of methods of collecting inocula of Meloidogyne spp., including a new technique. Plant Dis. Rptr. 57:1025-1028.

Noe, J.P. 1985. Analysis and interpretation of data from nematological experiments, p. 187-196. In: K. Barker, C.C. Carter, and J.N. Sasser (eds.). An advanced treatise on Meloidogyne. vol. 2. Methodology. N.C. State Univ. Graphics, Raleigh.

Sasser, J.N., C.C. Carter, and K.M. Hartman. 1984. Standardization of host suitability studies and reporting of resistance to root-knot nematodes. Crop Nematode Res. Control Project N.C. State Univ., U.S. Agency for Intl. Dev., Raleigh.

Thies, J.A. and R.L. Fery. 1998. Modified expression of the $N$ gene for southern root-knot nematode resistance in pepper at high soil temperatures. J. Amer. Soc. Hort. Sci. 123:1012-1015.

Thies, J.A. and R.L. Fery. 2000a. Characterization of resistance conferred by the $N$ gene to Meloidogyne arenaria races 1 and 2, M. hapla, and $M$. javanica in two sets of isogenic lines of Capsicum annuum L. J. Amer. Soc. Hort. Sci. 125:71-75.

Thies, J.A. and R.L. Fery. 2000b. Heat stability of resistance to Meloidogyne incognita in Scotch Bonnet peppers (Capsicum chinense Jacq.). J. Nematol. 32:356-361.

Thies, J.A., J.D. Mueller, and R.L. Fery. 1998. Use of a resistant pepper as a rotational crop to manage southern root-knot nematode. HortScience 33:716-718. 